\title{
Superconducting Order Parameter Nucleation and Critical Currents in the Presence of Weak Stray Fields in Superconductor/Insulator/Ferromagnet Hybrids
}

\author{
Vasiliy N. Kushnir ${ }^{1,2}$, Serghej L. Prischepa ${ }^{1,3, * \mathbb{D}}$, Michela Trezza ${ }^{4}$, Carla Cirillo ${ }^{5}$ (D) and Carmine Attanasio ${ }^{4,5}$ (D) \\ 1 Department of Telecommunications, Belarusian State University of Informatics and Radioelectronics, \\ 220013 Minsk, Belarus; vnkushnir@gmail.com \\ 2 Department of Theoretical Physics and Astrophysics, Belarusian State University, 220030 Minsk, Belarus \\ 3 Department of Condensed Matter Physics, National Research Nuclear University (MEPhI), \\ 115409 Moscow, Russia \\ 4 Department of Physics "E.R. Caianiello", Università degli Studi di Salerno, I-84084 Fisciano (Sa), Italy; \\ michelatrezza@gmail.com (M.T.); cattanasio@unisa.it (C.A.) \\ 5 Department of Physics “E.R. Caianiello", CNR-SPIN, c/o Università degli Studi di Salerno, \\ I-84084 Fisciano (Sa), Italy; carla.cirillo@spin.cnr.it \\ * Correspondence: prischepa@bsuir.by
}

Citation: Kushnir, V.N.; Prischepa, S.L.; Trezza, M.; Cirillo, C.; Attanasio, C. Superconducting Order Parameter Nucleation and Critical Currents in the Presence of Weak Stray Fields in Superconductor/Insulator/

Ferromagnet Hybrids. Coatings 2021, 11, 507. https://doi.org/10.3390/ coatings11050507

Received: 8 March 2021

Accepted: 21 April 2021

Published: 25 April 2021

Publisher's Note: MDPI stays neutral with regard to jurisdictional claims in published maps and institutional affiliations.

Copyright: (c) 2021 by the authors. Licensee MDPI, Basel, Switzerland. This article is an open access article distributed under the terms and conditions of the Creative Commons Attribution (CC BY) license (https:/ / creativecommons.org/licenses/by/ $4.0 /)$.

\begin{abstract}
The stray fields produced by ferromagnetic layers in Superconductor/Insulator/Ferromagnet (S/I/F) heterostructures may strongly influence their superconducting properties. Suitable magnetic configurations can be exploited to manipulate the main parameters of the hybrids. Here, the nucleation of the superconducting phase in an external magnetic field that periodically oscillates along the film width is studied on the base of the numerical solution of the linearized system of Usadel equations. In addition, the effect of the magnetic configuration of the F-layer on the temperature dependence of the critical current density, $J_{\mathrm{c}}(T)$, is investigated in the framework of the GinzburgLandau phenomenological theory on the base of the oscillating model of a stray field. By following this approach, the $J_{\mathrm{C}}(T)$ dependence of a Nb/SiO $2 / \mathrm{PdNi}$ trilayer is reproduced for different magnetic configurations of the PdNi layer.
\end{abstract}

Keywords: S/F hybrids; electromagnetic coupling; theories and models of superconducting state

\section{Introduction}

The literature devoted to the investigation of the interplay between superconductivity and ferromagnetism in artificial heterostructures is very rich, due to both the fundamental and applicative interest to Superconductor (S)/Ferromagnet (F) hybrids [1-4]. One of the most interesting problems concerns the role of the stray fields produced by the F-layers on the superconducting properties of S/F samples [1-3,5]. In fact, due to the influence of an inhomogeneous external magnetic field on the superconducting order parameter, a new class of phenomena in the physics of superconductors has been observed. On the other hand, the spatial inhomogeneity of the stray fields, generated by a ferromagnet located in intimate contact with a superconductor, represents a challenging problem in describing the superconducting properties of such S/F systems.

In this regard, the first experiments [1,2,6-10] focused on the study of the Abrikosov vortex-lattice and its pinning properties in a superconducting film subjected to the nonuniform stray field produced by either a system of magnetic inclusions (particles, dots, nanorods, etc.) or an F-layer [11-22]. More recently, the properties of proximity-coupled $\mathrm{S} / \mathrm{F}$ hybrids have been studied in a great detail and superconducting spintronic devices have been proposed [1,3,23-31]. One of the main ingredients to control the superconducting state in these systems is the change of the direction and/or amplitude of the magnetization 
of the F-layer. In this tuning process, demagnetized and remanence (both in- and out-ofplane) states may cause unaccounted effects $[27,28]$.

Moreover, the presence of an inhomogeneous magnetic field, as the one due to stray fields, makes it difficult to solve the problem of the nucleation of the superconductivity in a thin film. This task was studied in S/I/F systems (here "I" indicates an insulating layer). In most of the works, the theoretical investigation was performed in the framework of the Ginzburg-Landau (GL) theory [2,32-42] (except in the case of reference [36], where the possibility of triplet superconductivity induced by the domain walls was considered). One of the most interesting results was the prediction of maxima in the $T_{\mathrm{c}}$ as a function of the external homogeneous magnetic field $H_{0}$. These maxima can be asymmetric for different orientations of the applied field with respect to the plane of the sample. Apart from this, the location (relative to the domain wall) and the shape of the superconducting nuclei was studied [38]. In the case of stripe domains, when the field close to the ferromagnet can be approximated by a step function, the nucleation in the S-film is similar to the case of S/N structures ( $\mathrm{N}$ is a normal metal) [43-45].

Another problem, which received great attention, was the study of the critical current density, $J_{c}$, of a superconductor in the presence of magnetic interactions. In particular, the increase of $J_{\mathrm{c}}$ due to the magnetic pinning of vortices was analyzed. Peaks in the $J_{\mathrm{c}}\left(H_{0}\right)$ dependence due to the matching effect, predicted in reference [46], were observed in numerous experiments $[2,9,10,16,19]$. These data, along with the numerical analysis performed in [47], proved the ability to influence the critical current in a controlled manner. Moreover, measurements of the critical currents in layered S/I/F [48] and S/F [49] hybrids demonstrated a nontrivial dependence of $J_{\mathrm{c}}$ on the magnetic state of the ferromagnet, especially at temperatures significantly smaller than $T_{\mathrm{c}}$.

From the theoretical point of view, it is worth emphasizing that the presence of an inhomogeneous magnetic field, $\mathbf{H}(\mathbf{r})$, makes it extremely difficult to both formulate and solve the problems related to the properties of the vortex matter, the nucleation of the superconducting phase at a critical field $\mathbf{H}_{\mathrm{c}}(\mathbf{r})$, and the vortex-free state at weak magnetic fields.

In this paper, we consider two problems related to the critical parameters of a superconductor in a non-uniform magnetic field in a S/I/F hybrid. The first one is related to the nucleation of the superconductivity and the second concerns the study of the temperature dependence of the critical current density, $J_{\mathrm{c}}(T)$, in a vortex-free superconducting film. The problem of the nucleation of the superconductivity in the presence of an inhomogeneous stray field is formally considered as the problem of the upper critical amplitude of an "elementary" external inhomogeneous field. The strength of the field is assumed to have a sinusoidal shape. This kind of approach was already proposed and investigated experimentally in reference [32] in the framework of the GL theory for two limiting cases, i.e., for great and small amplitudes of the magnetic field. In reference [34] the GL equations were solved numerically in the case of a uniform and periodical field, modelled by a step-like periodic function. A two dimensional problem was solved within the framework of the phenomenological GL theory in reference [35] assuming a periodic repetition of a solution for the stray field of a domain wall [33]. In this work, we solve the problem for an elementary periodic model of an inhomogeneous magnetic field in the framework of the microscopic theory of superconductivity applying the formalism of the Usadel equations. In our case, the results are valid for the entire temperature range, from $T_{\mathrm{C}}$ down to $0 \mathrm{~K}$. The second task is related to the determination of the $J_{\mathrm{C}}(T)$ dependence in the case of an $\mathrm{S} / \mathrm{I} / \mathrm{F}$ hybrid where $\mathrm{F}$ is a weak ferromagnet and I is a sufficiently thick layer. Therefore, the magnitude of the stray field is weak enough to induce vortex nucleation in the superconductor. The configuration of the stray field was experimentally varied by applying an appropriate magnetizing/demagnetizing procedure which creates in- or out-of-plane remanence states (IPR- and OPR-states, respectively), as well as fully demagnetized state (D-state). We demonstrate that in all the magnetic states the spatial profile of the stray fields can be described by a smooth function that can be well approximated by several terms of 
a Fourier series. This justifies the use of the proposed elementary model for qualitative estimations. Under these assumptions, we were able to qualitatively explain the difference in the measured $J_{\mathrm{c}}(T)$ dependencies for different magnetic states in the framework of the GL formalism.

\section{Experimental}

In the $\mathrm{S} / \mathrm{I} / \mathrm{F}$ system under study the superconducting and ferromagnetic layers of equal thickness $d_{S}=d_{F}=30 \mathrm{~nm}$ are separated by a 20-nm-thick $\mathrm{SiO}_{2}$ film. Here, PdNi is a dilute ferromagnetic alloy with composition $\mathrm{Pd}_{84} \mathrm{Ni}_{16}$, and $\mathrm{Nb}$ was used as a superconductor. The heterostructure is deposited on a $\mathrm{Si}(100)$ substrate by sputtering technique in three different steps. First, a layer of $\mathrm{Nb}$ was sputtered on a silicon substrate by dc magnetron. Then the $\mathrm{SiO}_{2}$ barrier was realized by RF sputtering (MRC 8602), breaking the vacuum. Afterwards, the final PdNi layer was deposited by dc sputtering [49,50]. A sketch of the resulting substrate $/ \mathrm{Nb} / \mathrm{SiO}_{2} / \mathrm{PdNi}$ trilayer is shown in the inset of Figure 1 . Finally, the sample was patterned by lift-off into a strip of width $w=20 \mu \mathrm{m}$ and length $l=300 \mu \mathrm{m}$. All the transport measurements were performed in a ${ }^{4} \mathrm{He}$ cryostat by following the same procedure described in reference [49]. The $J_{\mathrm{C}}$ values were determined applying the $3 \mathrm{mV} / \mathrm{m}$ electric field criterion. The critical temperature of the trilayer is $T_{\mathrm{c}} \approx 4.5 \mathrm{~K}$, smaller when compared to the corresponding proximized $\mathrm{Nb} / \mathrm{PdNi}$ hybrid investigated in reference [49] $\left(T_{\mathrm{c}} \mathrm{Nb} / \mathrm{PdNi} \approx 6 \mathrm{~K}\right)$. This result can be attributed to the vacuum breaking during the deposition and the lift-off procedure. The estimated low-temperature resistivity of the $\mathrm{Nb}$ film is $\rho \approx 20 \mu \Omega \cdot \mathrm{cm}$, while the superconducting coherence length is $\xi=\sqrt{\hbar D / 2 \pi k_{B} T_{c}} \sim 9-10 \mathrm{~nm}[51]$, which results in a Ginzburg-Landau (GL) coherence length at $T=0 \xi_{G L}(0)=(\pi \xi / 2) \sim 14-16 \mathrm{~nm}$. This means that the ratio $d s / \xi_{G L}(T)$ varies in the range 1-1.6 over the entire temperature range of measurements, so that the $\mathrm{Nb}$ layer can be considered as a thin film. $J_{\mathrm{c}}$ was measured as a function of the temperature for three different magnetic configurations of the PdNi layer. The demagnetized state (D) is reached by cooling the as-grown sample from room temperature without any applied magnetic field. The IPR (OPR) state is obtained by first warming the sample above the PdNi Curie temperature, $T_{\text {Curie }} \approx 190 \mathrm{~K}$ [49], then cooling it down to $T=4.2 \mathrm{~K}$, and later applying a parallel (perpendicular) magnetic field well above the saturation field in order to magnetize the PdNi layer. Finally, the field is switched off. $J_{c}(T)$ is always measured in the absence of the magnetic field. In Figure 1 we show the dependence of $J_{\mathrm{c}}$ as a function of $T$ measured for the three magnetic states. It follows that the $J_{\mathrm{C}}(T)$ dependencies coincide for all states from $T_{\mathrm{c}}$ down to $T \approx 4 \mathrm{~K}$. In addition, only a slight difference can be observed between the critical current densities in the D and IPR states for lower temperatures, while the OPR state is characterized by more reduced values of $J_{\mathrm{c}}$. This result is radically different from the one reported in reference [49], where a pronounced dependence of the $J_{c}(T)$ values for the three different configurations was observed. In particular, the relation $J_{\mathrm{c}}{ }^{\mathrm{OPR}}<J_{\mathrm{c}}{ }^{\mathrm{IPR}}<J_{\mathrm{c}}{ }^{\mathrm{D}}$ was fulfilled in the entire measured temperature range. 


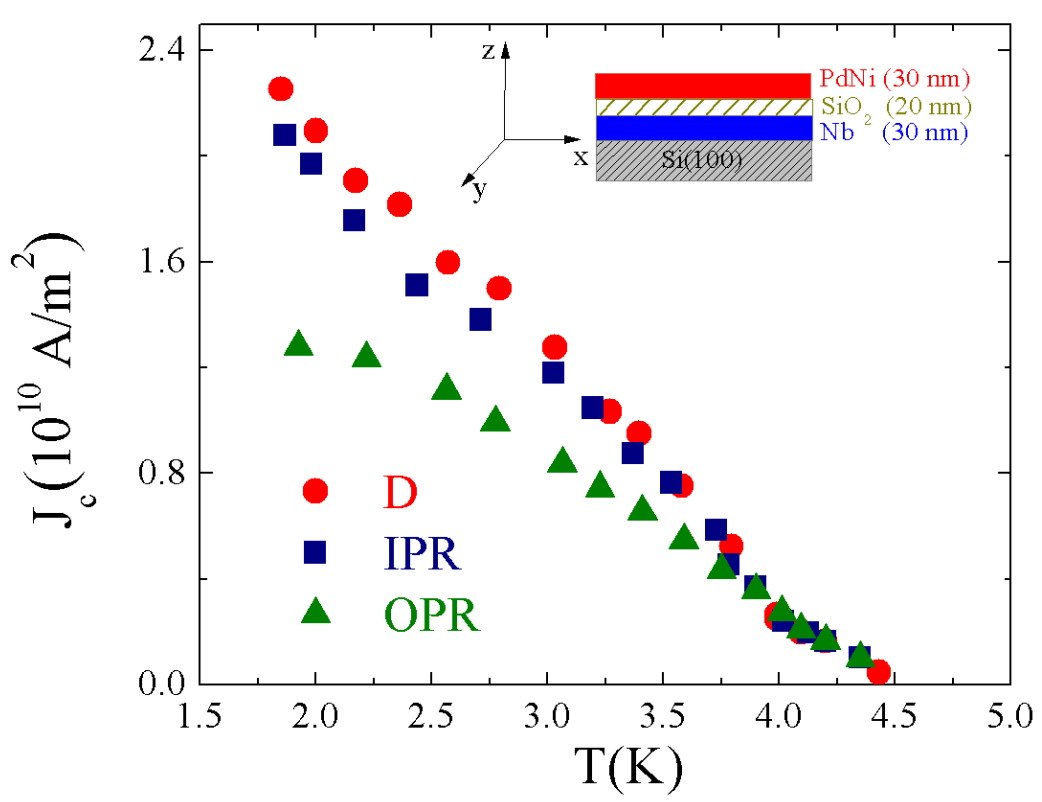

Figure 1. Critical current density as a function of the temperature for the three different magnetic configurations described in the text. Inset: schematic representation of the sample under study.

\section{Stray Fields of PdNi}

Before analyzing the problem of the nucleation of the superconductivity in the presence of an inhomogeneous magnetic field, we focus on the magnetic properties of the PdNi layer and the produced stray fields. From the magnetization measurements presented in reference [52] it is $\mu_{0} M_{\mid \text {I sat }} \sim 0.15 \mathrm{~T}$ and $\mu_{0} M_{\perp \text { sat }} \sim 0.2 \mathrm{~T}$, where $M_{||(\perp) \text {,sat }}$ is the in-plane (out-of-plane) saturation magnetization. Moreover, according to the data given in references [49] and [53] concerning the magnetocrystalline anisotropy of $\mathrm{Pd}_{81} \mathrm{Ni}_{19}$ and $\mathrm{Pd}_{88} \mathrm{Ni}_{12}$ films, respectively, we can assume that in the IPR (OPR) state of PdNi layer of the heterostructure under study it is $\mu_{0} M_{|| \text {,rem }} \approx 30 \% \mu_{0} M_{\mid \text {| sat }}\left(\mu_{0} M_{\perp, \text { rem }} \approx 70 \% \mu_{0} M_{\perp \text {,sat }}\right)$. As far as the domain structure of the PdNi film is concerned, it is widely assumed that in the $\mathrm{D}$ state the PdNi layer is arranged in randomly oriented domains of average diameter of about $d_{d} \approx 100 \mathrm{~nm}$, with a domain wall size not larger than $d_{w} \approx 10 \mathrm{~nm}$ [53]. Moreover, the perpendicular magnetic anisotropy (PMA) of thin PdNi films [49,53] implies the out-ofplane magnetization of domains in the D state. Perpendicular magnetization of domains is typical for thin films of ferromagnets with PMA [54-58]. This information is needed to estimate the distribution of the (orthogonal) $z$-component of the induction field, $B_{z}$, of the F-layer in the D, OPR, and IPR states. Therefore, for simplicity we assume a periodic domain lattice $[59,60]$ in agreement with what reported for another weak ferromagnetic alloy with out-of-plane magnetization, such as $\mathrm{Cu}_{47} \mathrm{Ni}_{53}$ [61]. The calculated distribution of $B_{\mathrm{Z}}(x)$ is shown in Figure 2 for the profile $y=0$ for some representative values of $z$. In particular, the dark yellow lines show the field on the surface of the ferromagnet $(z=30 \mathrm{~nm})$, namely the stray field present at the S/F interface in proximity coupled systems. It is evident that in this case the stray fields are rather intense $(\sim 100 \mathrm{mT})$ with a large gradient at the domain edges. The other simulations refer to other characteristic values of $z$, namely at the $\mathrm{SiO}_{2} / \mathrm{Nb}$ interface $(z=50 \mathrm{~nm})$, and in the middle (top surface) of the $\mathrm{Nb}$ layer $[z=65 \mathrm{~nm}(z=80 \mathrm{~nm})]$ (see inset of Figure 1). 

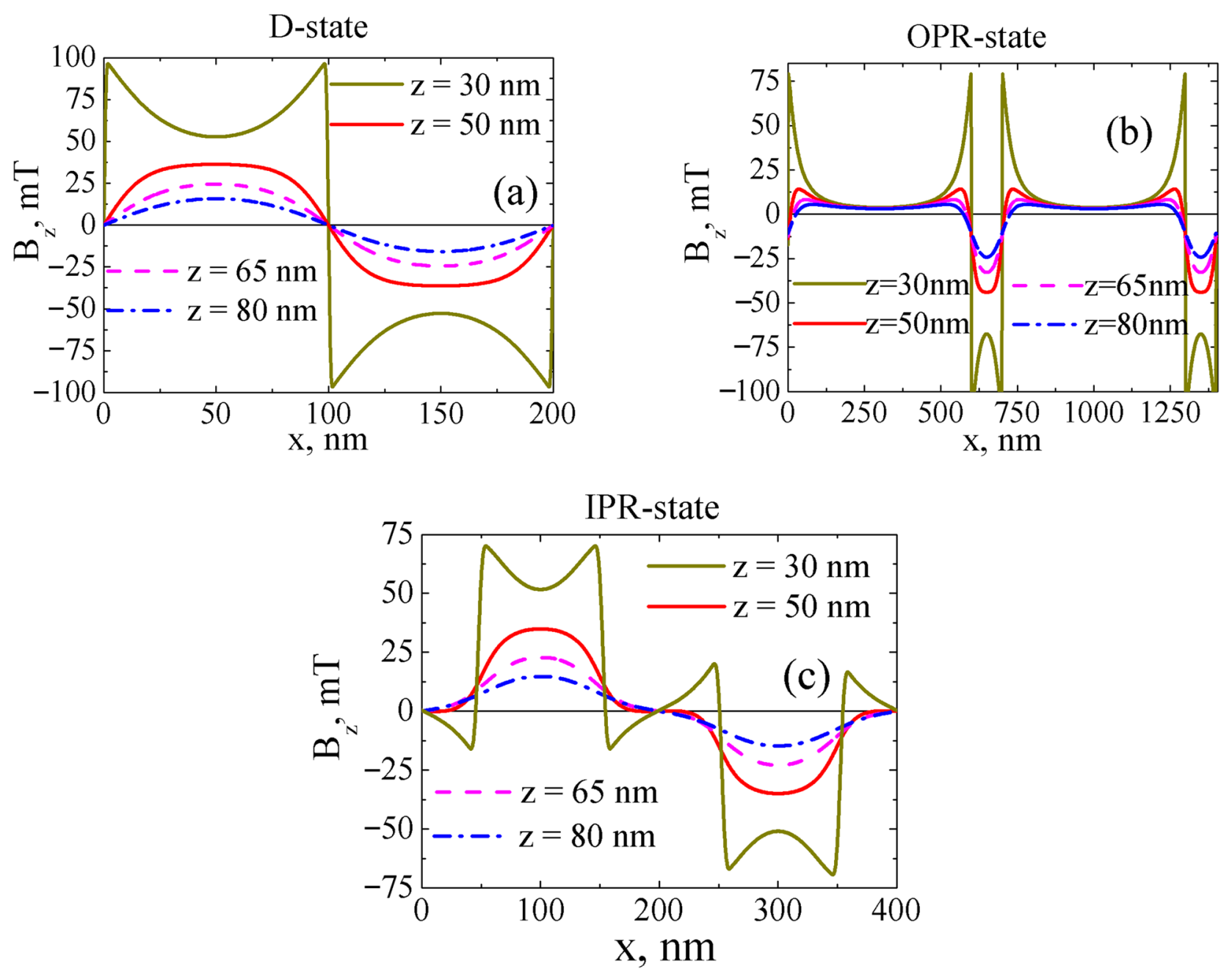

Figure 2. Distribution of the orthogonal component of the stray magnetic field $B_{z}$ versus $x$ for $y=0$ in the D (a), OPR (b) and IPR (c) states of the PdNi film and for different values of $z$.

In the D state, the amplitude of the stray field decreases with distance from the ferromagnetic layer from $36.5 \mathrm{mT}$ at $z=50 \mathrm{~nm}$ (at the $\mathrm{SiO}_{2} / \mathrm{Nb}$ interface) to $25.0 \mathrm{mT}$ at $z=65 \mathrm{~nm}$ (namely in the middle of the Nb layer) and $16.5 \mathrm{mT}$ at $z=80 \mathrm{~nm}$. The field strength in the OPR state is on average about $4 \mathrm{mT}$ over positive domains and varies in amplitude from $24 \mathrm{mT}$ to $44 \mathrm{mT}$ over negative domains. In the IPR state, regions with sufficiently large field values alternate with regions of the same size with a zero $z$-component of the field. In this configuration the amplitude of the stray fields is $35 \mathrm{mT}$ at $z=50 \mathrm{~nm}, 23 \mathrm{mT}$ at $z=65 \mathrm{~nm}$, and $15 \mathrm{mT}$ at $z=80 \mathrm{~nm}$. Some important observations can be mentioned. The $\mathrm{D}$ and IPR states are both characterized by $d_{d} \approx 100 \mathrm{~nm}$ and symmetric field distributions, i.e., for a regular domain structure, each region with a positive field corresponds to exactly the same region with a negative field. On the contrary, in the OPR state the dimensions of the domains depend on the field polarity, with larger positive domains of about $d_{d} \approx 600 \mathrm{~nm}$. Here, the stray field is very weak over positive domains and sharply increases in magnitude in the region of negative domains. Moreover, from the calculations it emerges that the presented characteristic values of the stray fields weakly depend on the distribution of domains in the XOY plane (this is explained by the fact that the structure is effectively infinite in the OX and OY directions). Finally, from the simulations it emerges that the periodicity of the magnetic profiles changes in the different states, the period, $d$, being 200, 400, and $700 \mathrm{~nm}$ in the D, IPR, and OPR states, respectively.

In the following, we consider the values of the stray fields experienced by the superconductor. It is worth noticing that our case differs from those considered in the literature 
since here the insulating layer which separates the $S$ and $F$ ones is rather thick $\left(d_{I}=20 \mathrm{~nm}\right)$, and of the order of $d_{F}$. As a result, the stray fields are significantly weakened in the S-layer. We empirically determine the characteristic values of $B$ for each magnetic configuration as the maximum value assumed by the stray field in the middle of the $S$ layer. For instance, $B_{z, \mathrm{OPR}_{+}} \sim 8 \mathrm{mT}\left(B_{\mathrm{z}, \mathrm{OPR}-} \sim 30 \mathrm{mT}\right)$ is the maximum value of the field induction in the middle of the $\mathrm{S}$ layer over "positive" ("negative") domains in the OPR state, while $B_{Z, \mathrm{D}} \sim B_{\mathrm{Z}, \mathrm{IPR}} \sim 20 \mathrm{mT}$ is the stray field strength in the $\mathrm{D}$ and the IPR states. The first question here is, how strongly does such a field depress $T_{\mathrm{c}}$ ? By following reference [34], the shift in $T_{\mathrm{c}}$ due to the "orbital effect", namely the difference between the critical temperature at zero field and the one in the presence of the stray field, can be expressed as $\Delta T_{\mathrm{C}}{ }^{\text {orb }}=T_{\mathrm{CO}}-T_{\mathrm{C}}=T_{\mathrm{Co}}\left(\xi_{\mathrm{GL}}(0) / L\right)^{2}$, where a characteristic length $L=\left(\Phi_{0} / 2 \pi B_{0}\right)^{1 / 2}$ is introduced. For the above-mentioned configurations, it is $L_{\mathrm{OPR}+} \sim 200 \mathrm{~nm}, L_{\mathrm{OPR}-} \sim 105 \mathrm{~nm}$, and $L_{\mathrm{D}} \sim L_{\mathrm{IPR}} \sim 130 \mathrm{~nm}$. Therefore, by considering an average stray field value of about $30 \mathrm{mT}$ and $B_{c 2}(T) \sim 3 \mathrm{~T}$, the largest critical temperatures suppression is $\Delta T_{\mathrm{c}} \sim T_{\mathrm{c}} \cdot 0.01<0.05 \mathrm{~K}$ for our system. Summarizing, since the stray fields are significantly smaller than the upper critical field, it results that the "orbital effect" is negligible here, as it can be inferred from Figure 1, where the $J_{\mathrm{C}}(T)$ dependence for the three configurations goes to zero at the same temperature.

\section{Superconductivity in Presence of Inhomogeneous Magnetic Fields}

This section is devoted to the influence of the magnetic fields previously derived on the superconducting state. It is known that the distribution of the stray field for a ferromagnetic slab is determined by the relation between its thickness $d_{F}$ and the distance between the domain walls $[2,34,62]$. When $d_{w}<<d_{F}<<d_{d}$, the resulting distribution produces only a local suppression of the superconductivity in a region of the order of $d_{d}$ above the domain wall [62]. These conditions can be considered fulfilled in the case of the $\mathrm{Nb} / \mathrm{SiO}_{2} / \mathrm{PdNi}$ tri-layer under study. Indeed, we have just seen that the stray field in the superconductor region is quite weak and has small effect on the critical temperature. In the next section, we will investigate the problem of the critical magnetic field in presence of an oscillating stray field. We can name this new concept "critical inhomogeneous field".

\subsection{Stray Field Induced Distribution of the Superconducting Condensate}

As already mentioned in the introduction, the problem of the nucleation of the superconductivity in a periodic magnetic field was first formulated in reference [32] and further considered in $[34,35]$ on the base of the approximate solution of GL equations. Here, the same problem is formulated and solved in the framework of the Usadel equation formalism, which is valid over the entire temperature range from 0 to $T_{\mathrm{c}}$.

In the elementary model, the induction field is orthogonal to the surface of the film and changes along one of the planar axes (OX) with periodicity $d$ according to the oscillating law $B_{\mathrm{z}}=B(x, z) \approx B_{0} \cos (\pi x / d)$. This field is associated to the vector-potential $\mathbf{A}(\mathbf{r})=\left(0, A_{y}(x), 0\right)$, where $A_{y}(x)=\left(B_{0} d / \pi\right) \sin (\pi x / d)$. This model is not only formal. Indeed, such a periodic field can be created by a stripe periodic domain structure with perpendicular magnetic anisotropy and alternating signs of the magnetic moments of stripes of width $d[11-13,63]$. However, it is obvious that this model is not suitable to describe the magnetic stray fields generated in the OPR state. Indeed, in this case, due to the relatively strong remanence, the magnetic profile of a domain strip is quite complex, namely, the periodic function $B(x)$ cannot be well approximated even considering several terms of the Fourier series (see Figure 2). The critical state of the superconductivity of the film is derived in the diffusive limit within the formalism of a linearized system of Usadel equations for the anomalous Green's functions $F_{\omega}(\mathbf{r})\left(\hbar \omega=\pi k_{\mathrm{B}} T(2 n+1), \hbar\right.$ is the reduced Planck constant, $k_{\mathrm{B}}$ is the Boltzmann constant, $\left.n=0,1,2 \ldots\right)$ [64]. Given the expression of 
A(r) (see above), this system is reduced to the equation for a single function $\Psi(x / \xi)$ by the substitution $F_{\omega}(\mathbf{r})=c_{\omega} e^{i k y /} \Psi(x / \xi)$ :

$$
-\Psi^{\prime \prime}(\tau)+\left(k-\frac{B_{0}}{v B_{2}} \sin (v \tau)\right)^{2} \Psi(\tau)=\mu \Psi(\tau),
$$

where the scaled coordinate $\tau \equiv x / \xi$ and the scaled field $B_{2} \equiv \Phi_{0} / 2 \pi \xi^{2}$ are introduced. Furthermore, $k$ is the superfluid velocity parameter [65], $v=\pi \xi / d$ is the wave number, and $c_{\omega}$ are the coefficients that satisfy a homogeneous system of linear algebraic equations. The condition of the existence of a nontrivial solution of this system leads to the characteristic equation for $\mu$ :

$$
\psi\left(\frac{1}{2}+\frac{\mu T_{S}}{2 T}\right)-\psi\left(\frac{1}{2}\right)=\ln \left(\frac{T_{S}}{T}\right),
$$

where $\psi(z)$ is the digamma-function [66] and $T_{\mathrm{S}}$ is the critical temperature of the isolated film. The solution of Equation (2) is expressed by the De Gennes universal function $(T)=U n\left(T / T_{S}\right) /(2 \pi)$ [67], which monotonically decreases with $T$. Therefore, the problem of the largest (i.e., critical) temperature, for which a bounded solution of the Equation (1) exists, is equivalent to the problem of the minimum of $\mu$. Note that Equation (1) belongs to the class of Hill equations that have been studied in detail (see, e.g., reference [68]), so only the main results of the calculations and their physical interpretation are given here.

First of all, we reformulate the problem of the minimum of $\mu$ as the problem of the maximum of the amplitude $B_{0}$, which depends on three parameters, namely $v, T$ and $k$. Then, the following algorithm was used. For a given wave number $v$, we look for the maximum value of the parameter $B_{0}$, which we denote as $B_{0 \mathrm{~m}}(T ; k)$, since it depends on both $k$ and $T$. Afterwards, for a fixed temperature we determine the $k$ value, which corresponds to the maximum of $B_{0 \mathrm{~m}}(T ; k)$. This will be the critical amplitude $B_{0 \mathrm{c}}(T)$. Thus, for each $v$ we have a family of $B_{0 \mathrm{~m}}(T ; k)$ curves from which we extract the characteristic $B_{0 c}(T)$. An example of such a calculation is shown in Figure 3 for $v=0.2$, a value comparable with the one calculated for our S/I/F system. In particular, Figure 3a shows the family of characteristics $B_{0 \mathrm{~m}}(T ; k)$ for fixed values of the reduced temperature $t=T / T_{\mathrm{S}}$ in the interval 0.01-0.9. Figure $3 \mathrm{~b}$ shows the extracted critical characteristics $B_{0 \mathrm{c}}(T)$ compared to the dependence obtained for $k=0$ (the physical meaning of this value will be explained below), denoted as $B_{0 \mathrm{~m}}(T ; k=0)$.
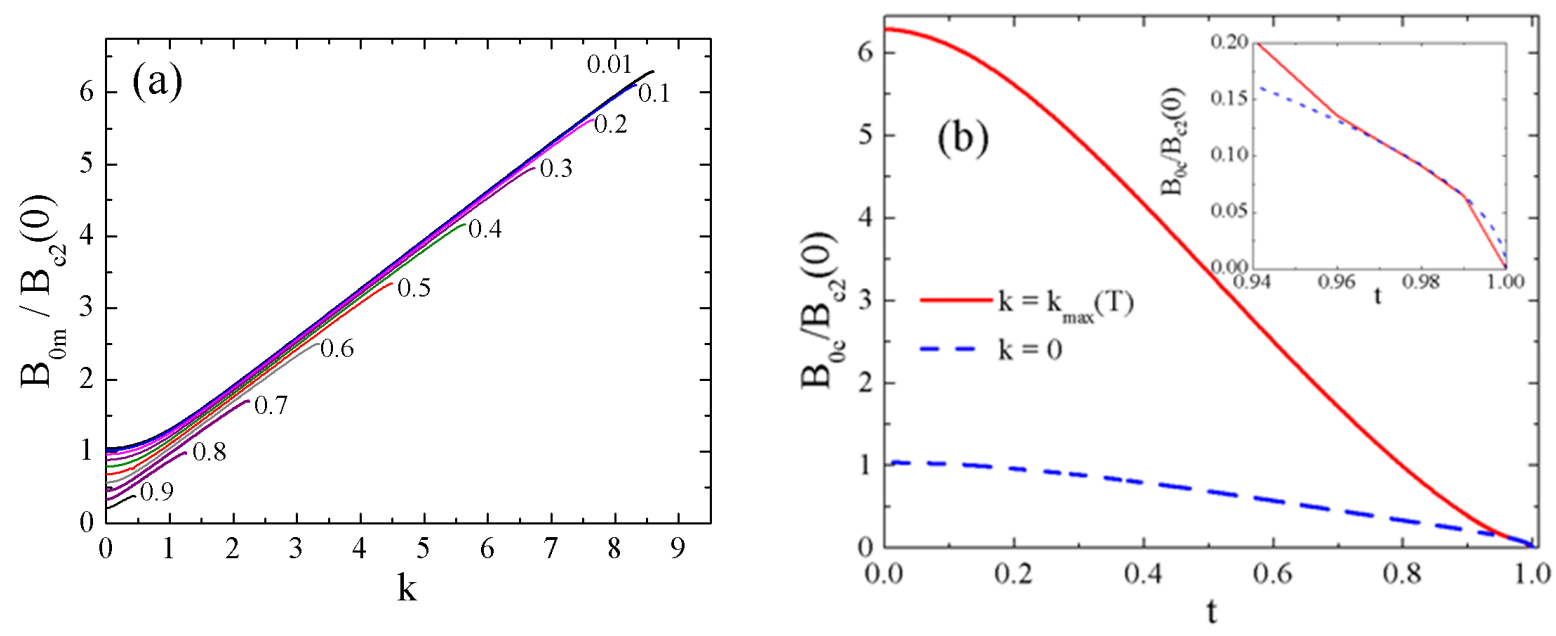

Figure 3. Dependence of the scaled critical amplitude of the magnetic field for $v=0.2$ on (a) the superfluid velocity parameter $k$ at various reduced temperatures $t=T / T_{S}$ reported as a label, (b) on the reduced temperature $t$ for $k=0$ and $k_{\max }(T)$. Inset shows the enlarged region close to $T_{\mathrm{S}}$. 
The characteristic obtained in the particular case $v=0.2$, reflects the form of the dependence of $B_{0 \mathrm{c}}(T)$ obtained also for other values of the wave number $v$. At the same time, by considering the left side of Equation (1), we can formulate the following statements about two limiting behaviors:

(i) for $v \rightarrow \infty(d \rightarrow 0)$ the influence of the magnetic field disappears;

(ii) in the limit $d \rightarrow \infty(v \rightarrow 0)$, for arbitrary $\tau<<v^{-1}$ the periodic function in Equation (1) becomes linear. This means that the dependence $B_{0 \mathrm{~m}}(T ; k=0)$ merges with the characteristic $B_{\mathrm{c} 2}(T)$ (the upper critical field of an isolated film). This limit is reached for $d \gtrsim 2 \pi \xi(\nu \lesssim 0.5)$.

Moreover, important considerations can be derived by analyzing Figure 3b. First, the critical field $B_{0 c}(T)$ is significantly larger than the upper critical field $B_{\mathrm{c} 2}(T)$ over almost the entire temperature range. This result demonstrates that the definition of the critical parameters in a nonhomogeneous magnetic field is not trivial. Next, from Figure $3 \mathrm{~b}$ we see that the critical state corresponds to $k_{\max }(T) \neq 0$ in the same $T$ range. Finally, only in the immediate vicinity of $T_{\mathrm{S}}$, the value $k=0$ gives the maximum to the function $B_{0 \mathrm{~m}}(T ; k)$, and this maximum almost coincides with $B_{\mathrm{c} 2}(T)$. This result is presented in the inset of Figure $3 \mathrm{~b}$. In this temperature range our results almost coincide with the ones of reference [34]. It is clear in view of the fact that in the latter case (i.e., in the region of the applicability of the GL theory) and for small values of $v$, Equation (1) reduces to the equation for the upper critical magnetic field.

The process of nucleation becomes clear as soon as we consider the "evolution" of the condensate wave function $\Psi(x)$ while moving along the $B_{0 m}(T ; k)$ curve at a fixed temperature.

In Figure 4 we show the functions $\Psi(x)$ calculated at the same $v=0.2$ and for the reduced temperature $t=0.92$ for different values of $k$ from zero up to $k_{\max }$.

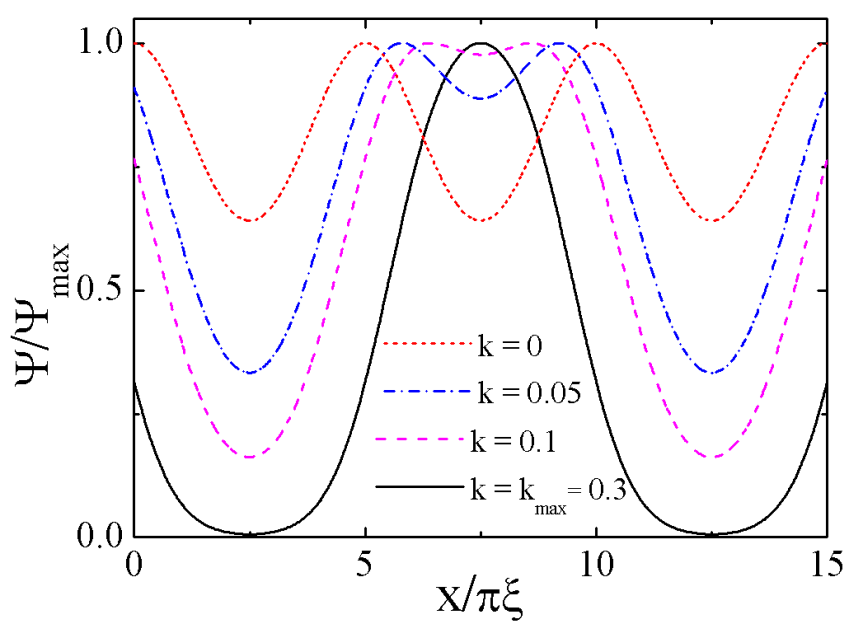

Figure 4. Superconducting wave function of a film in the presence of a magnetic field, $B_{0}(k) \cos (x / 5 \xi)$, for different values of the superfluid velocity parameter $k$.

As follows from Figure 4, as soon as the superfluid velocity parameter $k$ becomes non-zero, the topology of the wave function changes. Namely, from being $d$-periodic it becomes $2 d$-periodic with two adjacent maxima. Further, as the parameter $k$ increases, the neighboring maxima of the wave function converge in pairs, with the minimum between them being smoothed out while the minimum between the pairs deepens. At last, at the critical point, $k_{\max }$, two neighboring maxima merge, and the minima between the pairs touch the abscissa axis. Therefore, we can say that a $2 d$ periodic lattice of superconducting phase nuclei is formed at the critical point. From a physical point of view, it means that, when the magnetic field amplitude increases above the value of $B_{\mathrm{c} 2}(T)$, the condensate acquires a phase multiplier $e^{i k y / \xi}$, with a corresponding redistribution of the superconducting 
current, to minimize the effect of the magnetic field on the superconducting nuclei; this is a general property of inhomogeneous superconductivity.

\subsection{Superconducting Critical Current of a Thin Film in a Periodic Magnetic Field}

Now we can qualitatively examine the experimental dependences $J_{c}(T)$ of Figure 1. Analyzing the experimental S/I/F structure, we assume an approximation of the uniform distribution of the condensate over the thickness of the unperturbed S-film. To validate this assumption, we calculate the scaled critical current density of the isolated $\mathrm{S}$ film, $j_{\mathrm{c}}$ (directed along the OY axis), the distribution of the magnetic field due to the current, $B_{\mathrm{x}}$, and the condensate wave function. As an example, Figure 5 demonstrates the result obtained by solving numerically one-dimensional GL equations (see, for example, reference [69]) with $\kappa=\left(\lambda / \xi_{\mathrm{GL}}\right)=10(\lambda$ is the magnetic field penetration depth $)$ and $d_{\mathrm{S}}=4 \xi_{\mathrm{GL}}$.
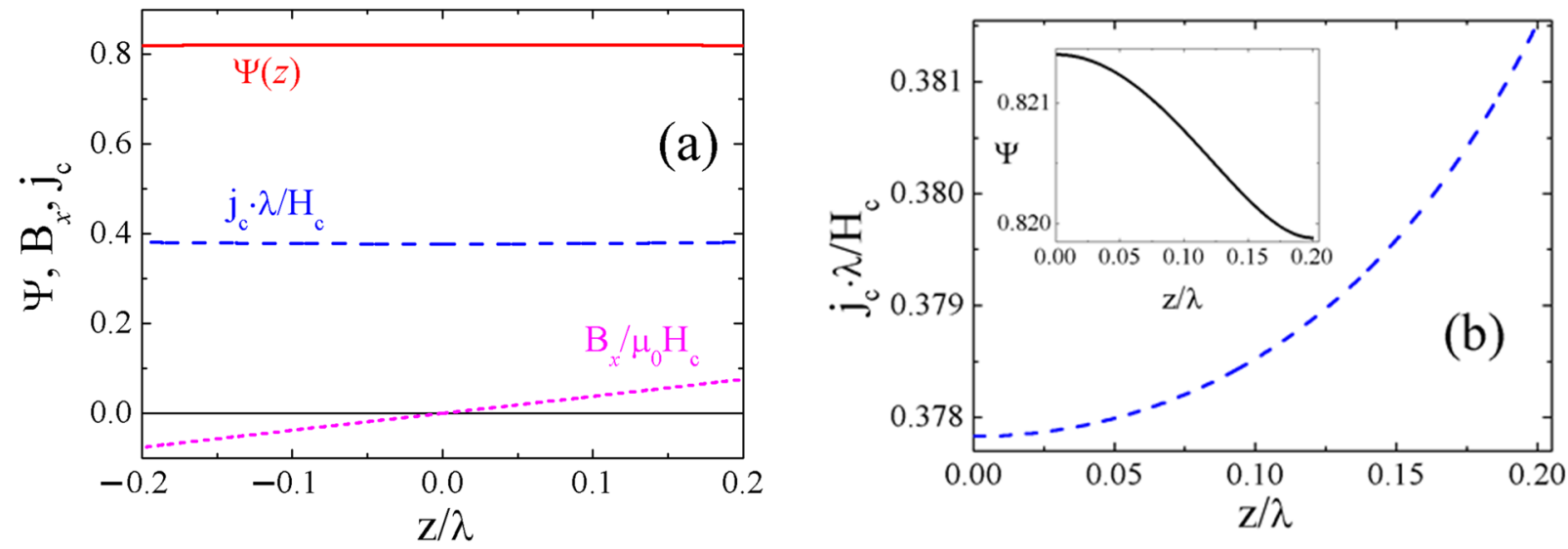

Figure 5. (a) The condensate wave function, $\Psi$, the critical current density, $J c$, and the magnetic field induction $B_{\mathrm{x}}$ in the S-film. (b) The current distribution in the $S$ film. Inset: the wave function $\Psi$ in the region $z>0$ on an enlarged scale. $H_{\mathrm{c}}$ is the thermodynamic critical field.

It follows from the Figure 5 that the total change in the condensate wave function does not exceed $0.5 \%$; therefore, the critical current density is determined by the classical formula of the GL theory. This confirms the validity of the approximation of a uniform condensate distribution for the considered $d_{\mathrm{S}}$ thicknesses. It is worth noting that as soon as we take into account the finite size of the film in the OX direction (perpendicular to the current), a complex question about the vortex phase immediately arises. In the following, we assume that the film is infinite in the OX direction and, consequently, edge effects are not taken into account [70].

Therefore, by following the oscillating model of the stray field, we can write the expression for the dimensionless current density $j$ of the GL model in the following form:

$$
j(\tau)=\kappa^{2}\left(k-\frac{B_{0}}{B_{c 2}(T) v_{G L}(T)} \sin \left(v_{G L}(T) \tau\right)\right) \Psi^{2}(\tau)
$$

Here, the normalized current density is defined as $j \equiv J / J_{\mathrm{sc}}$, where $J_{\mathrm{sc}}=B_{\mathrm{c} 2} /\left(\mu_{0} \xi_{\mathrm{GL}}\right)$. Further, contrary to Equation (1), the quantities $\tau, v_{\mathrm{GL}}$, are rescaled with respect to $\xi_{\mathrm{GL}}(T)$ and natural scale $B_{\mathrm{c} 2}(T)=\Phi_{0} / 2 \pi \xi_{\mathrm{GL}}{ }^{2}(T)$ is used for the critical magnetic field. (Now the wave number is expressed as $v_{\mathrm{GL}}(T)=\pi \xi_{\mathrm{GL}}(T) / d$.) Note that for $B_{0}=0$, as it is obvious, the classical law for the temperature dependence of the critical current density $J_{\mathrm{c}}(T) \sim\left(1-T / T_{\mathrm{c}}\right)^{3 / 2}[65,67,70]$ follows directly from Equation (3) by simply taking into account the temperature dependence of the used scales.

Now let us pay attention to the value of the ratio $b_{0} \equiv B_{0} / B_{\mathrm{c} 2}(T)$ in Equation (3). By using the values of the stray fields evaluated in Section 3, we obtain that $b_{0}$ is of the order of 0.01 at $T=0 \mathrm{~K}$. Furthermore, taking into account the previously given values $d \sim 100(200) \mathrm{nm}$ for the $\mathrm{D}$ (IPR) state and $\xi_{\mathrm{GL}}(0) \sim 15 \mathrm{~nm}$, we get $v_{\mathrm{GL}, \mathrm{D}}(0) \sim 0.5$ for the 
D state and $v_{\mathrm{GL}, I P R}(0) \sim 0.25$ for the IPR state (see the profiles distribution of the stray field in Figure 2). In addition, it should be emphasized that, according to reference [22], the low critical magnetic field is determined by the domain size which in our case is of the order of $100 \mathrm{~nm}$, namely smaller than $\lambda$ [49]. In other words, the average value of the stray field on the half-period is smaller than the low critical magnetic field. Therefore, the possibility of the vortex-antivortex pairs nucleation can be ignored. As a result, it reasonable to assume that almost in the entire temperature range, the stray fields weakly perturb both the order parameter modulation and the uniformly distributed current. For this reason the temperature dependence of the average measured critical current density, $\langle j\rangle$, can be evaluated by the variation principle for the GL functional [71] using a trial function $\tilde{\Psi}(\tau)=\psi_{0}+\left(b_{0} / v_{G L}\right) \psi_{1} \sin \left(v_{G L} \tau\right)$ (the term proportional to $\cos \left(v_{G L} \tau\right)$ vanishes). Substituting the derived constants $\psi_{0}, \psi_{1}$ in the expression for $j$, followed by the routine procedure of averaging and, further, maximizing $\langle j\rangle$ in the parameter $k$, with an accuracy of the third order in the parameter $\left(b_{0} / v_{\mathrm{GL}}\right)$, we finally get the expression in the usual measurement units.

$$
J_{\mathcal{c}}(T)=J_{0}\left(1-\frac{T}{T_{\mathcal{C}}}\right)^{3 / 2}\left[1-\varphi\left(v_{G L}(T)\right)\left(\frac{b_{0}(0)}{v_{G L}(0)}\right)^{2}\left(1-\frac{T}{T_{\mathcal{C}}}\right)\right]
$$

Here the constant $J_{0}$ is the critical current density of an isolated $S$ film at $T=0$. The positive quantity $\varphi\left(v_{G L}(T)\right)$ is determined by the expression $\varphi\left(v_{G L}\right)=\sum_{n=0,1,2} a_{n}\left(v^{2}+c_{0}^{2}\right)^{-n}$, where $a_{\mathrm{n}}$ and $c_{0}$ are numbers of order of unity. Since $\varphi\left(v_{G L}(T)\right)$ only moderately changes in the interval $T \in\left[0, T_{\mathrm{c}}\right]$ and the parameter $v_{\mathrm{GL}}{ }^{2}(0)$ is small, it is reasonable to assume $\varphi\left(v_{G L}(T)\right) \approx \varphi_{0}=$ const.

In accordance with Equation (4), for the relative difference $\Delta J_{c}(T)=J_{c, D}(T)-J_{c, I P R}(T)$ of critical currents $J_{\mathrm{c}, \mathrm{D}}(T)$ and $J_{\mathrm{c}, \mathrm{IPR}}(T)$ describing $\mathrm{D}$ and IPR states, respectively, we estimate $\Delta J_{c}(T) / J_{c, \mathrm{D}}(T) \approx 3 \varphi_{0} \cdot\left(b_{0} / v_{\mathrm{GL}, \mathrm{D}}\right)^{2}\left(1-T / T_{c}\right)$ (here $\left.v_{\mathrm{GL}, \mathrm{D}}=2 v_{\mathrm{GL}, \mathrm{IPR}}\right)$. The result of the fitting procedure to the Equation (4) for $J_{\mathrm{C}}(T)$ for two states, D and IPR, is shown in Figure 5. It results that the proposed model reproduces the overlap of the $J_{c, D}(T)$ and $J_{c, I P R}(T)$ curves close to $T_{\mathrm{c}}$ and their small difference at low temperatures, with the critical current density for the D state laying above the one for the IPR state, as expected. It is worth underlining that the quantitative agreement of the theoretical dependences of the critical current density with the corresponding experimental characteristics can be achieved by the implementation of a theoretical model based on the formalism of the Usadel equations. The meaning of the estimations of the $J_{\mathrm{c}}(T)$ is that the observed equality of $J_{\mathrm{c}}$ close to $T_{\mathrm{c}}$ and difference when the temperature decreases can be easily explained by the interplay between the $v_{\mathrm{GL}, \mathrm{D}}(0)$ and $v_{\mathrm{GL}, I P R}(0)$ on one side and the term $\left(1-T / T_{\mathrm{c}}\right)$ in Equation (4) on the other side. When $T$ tends to $T_{c}$, the difference between $v_{\mathrm{GL}, \mathrm{D}}(0)$ and $v_{\mathrm{GL}, I P R}(0)$ can be neglected in Equation (4), whereas this is not true at low $T$. Thus the morphology of the stray field enters the temperature dependence of the critical current density in S/I/F hybrids. It is necessary to underline that the observed effect could be important in the vortex-free state. In this regard, we pay attention on the $J_{\mathrm{c}, \mathrm{OPR}}(T)$ dependence in Figure 1 , which was not fitted in Figure 6. In this case, it is difficult to talk about the vortex-free state, since we have a fairly large size of the "positive" domains and the stray field over the negative domains is quite large. As a result, the elementary model is not applicable, i.e., the consideration of this case does not fit the format of this work. This issue, together with the full theoretical explanation of the $J_{\mathrm{c}, \mathrm{OPR}}(T)$ curve, will be the subject of a future work. 


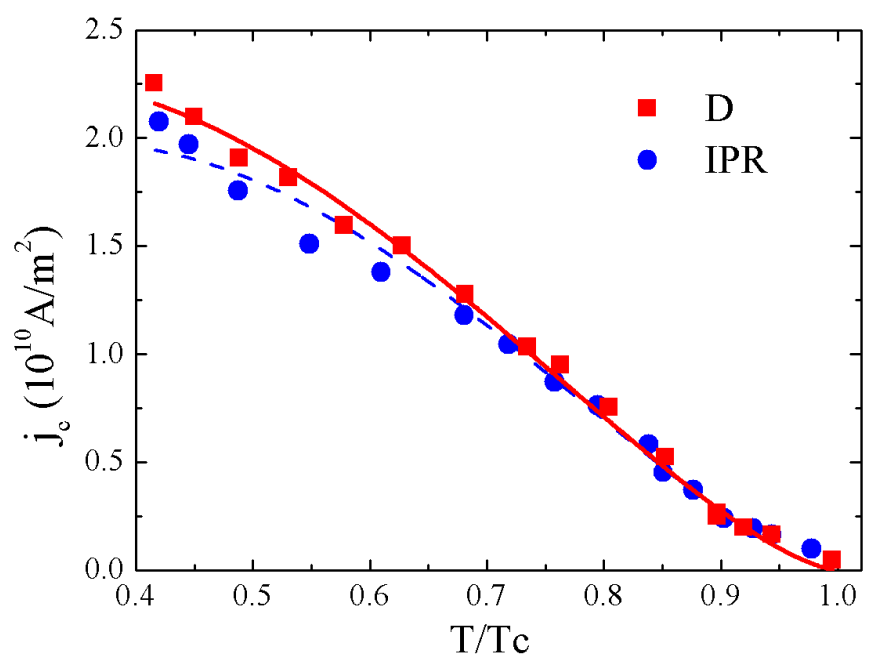

Figure 6. Critical current density as a function of $\mathrm{T} / \mathrm{T}_{\mathrm{c}}$ measured for the $\mathrm{Nb} / \mathrm{SiO}_{2} / \mathrm{PdNi}$ trilayer in the magnetic configurations labeled as D (squares) and IPR (circles). Lines are the results of the modeling for the corresponding states, indicated by thick solid red and thin dashed blue lines, respectively.

\section{Conclusions}

The nucleation of superconductivity in strongly nonuniform magnetic field is a challenging problem. Here we made a progress on the idealized case of weak sinusoidal magnetic field created by a thin ferromagnet layer in S/I/F hybrid. The I-layer thickness is such that the magnetic field created by a dilute F in the D- and IPR-states does not exceed the first critical magnetic field of superconductor. Thus we exclude the process of vortex-antivortex pair formation, which inevitably distorts the topology of the pair wave function. As a result, two problems were solved.

First, within the formalism of the Usadel equations we made accurate predictions for important properties of the critical amplitude of the non-uniform magnetic field and its temperature dependence. Only close to the critical temperature $B_{0 \mathrm{c}} \approx B_{\mathrm{c} 2}$ in thin S-film, whereas at $t<<1$ it is $B_{0 \mathrm{c}}>>B_{\mathrm{c} 2}$. This effect can be understood as a result of redistribution of the superconducting current in a strongly non-uniform magnetic field to minimize its effect on the superconducting nuclei.

Second, considering the same periodic modulation for the amplitude of the magnetic field, the qualitative explanations for the $J_{c}(T)$ dependencies for the D- and IPR-states have been provided in the framework of the GL theory. The equality of the critical currents near the $T_{\mathrm{c}}$ and lower $J_{\mathrm{c}}$ values for the IPR-state with respect to that for D-state at $T<<T_{\mathrm{c}}$ are explained through the difference in the period of the stray field modulation in these two states, and demonstrate that smaller period has a greater impact on $J_{\mathrm{c}}$.

On the base of these results, the perspectives of this work can be delineated. In particular, in the case of S/I/F systems with variable thickness of the different layers, the control of the magnetic state of the $\mathrm{F}$ layer may give important indications for the realization of samples with tunable $J_{c}(T)$. The suitable choice of both the thickness and the materials may help in the design of S/I/F switching devices. The approach we used is quite general and can be employed to understand other similar systems, such as S/F hybrids with a triplet component of the wave function, and provide a solid foundation on which even more complicated behaviors of the critical current and superconducting nuclei can be explained.

Author Contributions: V.N.K., S.L.P., C.C., and C.A. conceived the experiment. M.T. deposited the sample. M.T., S.L.P., and C.C. performed the transport measurements. V.N.K. elaborated the theoretical model and performed the calculations. V.N.K., S.L.P., C.C., and C.A. wrote the manuscript. All authors have read and agreed to the published version of the manuscript.

Funding: This research received no external funding. 
Institutional Review Board Statement: Not applicable.

Informed Consent Statement: Not applicable.

Data Availability Statement: Not applicable.

Acknowledgments: The authors gratefully acknowledge G. Carapella for depositing the $\mathrm{SiO}_{2}$ layer. S.L. Prischepa acknowledges the partial financial support of the "Improving of the Competitiveness" Program of the National Research Nuclear University MEPhI-Moscow Engineering Physics Institute.

Conflicts of Interest: The authors declare no conflict of interest.

\section{References}

1. Lyuksyutov, I.F.; Pokrovsky, V.L. Ferromagnet-superconductor hybrids. Adv. Phys. 2005, 54, 67-136. [CrossRef]

2. Aladyshkin, A.Y.; Silhanek, A.V.; Gillijns, W.; Moshchalkov, V.V. Nucleation of superconductivity and vortex matter in superconductor-ferromagnet hybrids. Supercond. Sci. Technol. 2009, 22, 053001. [CrossRef]

3. Buzdin, A.I. Proximity effects in superconductor-ferromagnet heterostructures. Rev. Mod. Phys. 2005, 77, 935-976. [CrossRef]

4. Golovchanskiy, I.A.; Abramov, N.N.; Stolyarov, V.S.; Dzhumaev, P.S.; Emelyanova, O.V.; Golubov, A.A.; Ryazanov, V.V.; Ustinov, A.V. Ferromagnet/superconductor hybrid magnonic metamaterials. Adv. Sci. 2019, 6, 1900435. [CrossRef]

5. Paschoa, A.; Gonzalez, J.L.; Nascimento, V.P.; Passamani, E.C. The role of the stray field on superconducting properties of hybrid ferromagnetic/superconducting heterostructures. J. Appl. Phys. 2020, 128, 043902. [CrossRef]

6. Geoffroy, O.; Givord, D.; Otani, Y.; Pannetier, B.; Ossart, F. Magnetic and transport properties of ferromagnetic particulate arrays fabricated on superconducting thin films. J. Magn. Magn. Mater. 1993, 121, 223-226. [CrossRef]

7. Otani, Y.; Pannetier, B.; Nozières, J.P.; Givord, D. Magnetostatic interactions between magnetic arrays and superconducting thin films. J. Magn. Magn. Mater. 1993, 126, 622-625. [CrossRef]

8. Nozaki, Y.; Otani, Y.; Runge, K.; Miyajima, H. Magnetic flux penetration process in two-dimensional superconductor covered with ferromagnetic particle array. J. Appl. Phys. 1996, 79, 8571-8577. [CrossRef]

9. Martín, J.I.; Vélez, M.; Nogués, M.J.; Schuller, I.K. Flux pinning in a superconductor by an array of submicrometer magnetic dots. Phys. Rev. Lett. 1997, 79, 1929-1932. [CrossRef]

10. Morgan, D.J.; Ketterson, J.B. Asymmetric flux pinning in a regular array of magnetic dipoles. Phys. Rev. Lett. 1998, 80, 3614-3617. [CrossRef]

11. Bulaevskii, L.N.; Chudnovsky, E.M.; Maley, E.P. Magnetic pinning in superconductor-ferromagnet multilayers. Appl. Phys. Lett. 2000, 76, 2594-2596. [CrossRef]

12. Bulaevskii, L.N.; Chudnovsky, E.M. Ferromagnetic film on a superconducting substrate. Phys. Rev. B 2000, 63, 012502. [CrossRef]

13. Bespyatykh, Y.I.; Wasilevski, W.; Gajdek, M.; Nikitin, I.P.; Nikitov, S.A. Pinning of vortices by the domain structure in a two-layered type II superconductor-ferromagnet system. Phys. Solid State 2001, 43, 1827-1833. [CrossRef]

14. Milošević, M.V.; Yampolskii, S.V.; Peeters, F.M. Magnetic pinning of vortices in a superconducting film: The (anti)vortex-magnetic dipole interaction energy in the London approximation. Phys. Rev. B 2002, 66, 174519. [CrossRef]

15. Vélez, M.; Martín, J.I.; Villegas, J.E.; Hoffmann, A.; González, E.M.; Vicent, J.L.; Schuller, I.K. Superconducting vortex pinning with artificial magnetic nanostructures. J. Magn. Magn. Mater. 2008, 320, 2547-2562. [CrossRef]

16. Milošević, M.V.; Peeters, F.M. Vortex-antivortex lattices in superconducting films with magnetic pinning arrays. Phys. Rev. Lett. 2004, 93, 267006. [CrossRef]

17. Helseth, L.E. Interaction between superconducting films and magnetic nanostructures. Phys. Rev. B 2002, 66, 104508. [CrossRef]

18. Zhu, L.Y.; Cieplak, M.Z.; Chien, C.L. Tunable phase diagram and vortex pinning in a superconductor-ferromagnet bilayer. Phys. Rev. B 2010, 82, 060503. [CrossRef]

19. Cieplak, M.Z.; Adamus, Z.; Kończykowski, M.; Zhu, L.Y.; Cheng, X.M.; Chien, C.L. Tuning vortex confinement by magnetic domains in a superconductor/ferromagnet bilayer. Phys. Rev. B 2013, 87, 014519. [CrossRef]

20. Bang, W.; Teizer, W.; Rathnayaka, K.K.D.; Lyuksyutov, I.F.; Naugle, D.G. Controlling superconductivity in thin film with and external array of magnetic nanostructures. Int. J. Mod. Phys. B 2015, 29, 1542035. [CrossRef]

21. Bang, W.; Teizer, W.; Rathnayaka, K.K.D.; Lyuksyutov, I.F.; Naugle, D.G. Using electrochemical fabrication to grow external arrays of magnetic nanostripes to manipulate superconductivity in the thin film. Int. J. Mod. Phys. B 2015, 29, 1542036. [CrossRef]

22. Lyuksyutov, I.F.; Pokrovsky, V.L. Magnetization controlled superconductivity in a film with magnetic dots. Phys. Rev. Lett. 1998, 81, 2344-2347. [CrossRef]

23. Golovchanskiy, I.A.; Abramov, N.N.; Stolyarov, V.S.; Chichkov, V.I.; Silaev, M.; Shchetinin, I.V.; Golubov, A.A.; Ryazanov, V.V.; Ustinov, A.V.; Kupriyanov, M.Y. Magnetization Dynamics in Proximity-Coupled Superconductor-Ferromagnet-Superconductor Multilayers. Phys. Rev. Appl. 2020, 14, 024086. [CrossRef]

24. Bergeret, F.S.; Volkov, A.F.; Efetov, K.B. Odd triplet superconductivity and related phenomena in superconductor-ferromagnet structures. Rev. Mod. Phys. 2005, 77, 1321-1373. [CrossRef]

25. Attanasio, C.; Maritato, L.; Prischepa, S.L.; Salvato, M.; Engel, B.N.; Falco, C.M. Superconducting properties of Nb-CuMn multilayers. J. Appl. Phys. 1995, 77, 2081-2086. [CrossRef] 
26. Kushnir, V.N.; Prischepa, S.L.; Cirillo, C.; Vecchione, A.; Attanasio, C.; Kupriyanov, M.Y.; Aarts, J. Multiple order parameter configurations in superconductor/ferromagnet multilayers. Phys. Rev. B 2011, 84, 214512. [CrossRef]

27. Kushnir, V.N.; Sidorenko, A.; Tagirov, L.R.; Kupriyanov, M.Y. Basic superconducting spin valves. In Functional Nanostructures and Metamaterials for Superconducting Spintronics (From Superconducting Qubits to Self-Organized Nanostructures), Springer Series Nano Science and Technology; Sidorenko, A., Ed.; Springer International Publishing: Cham, Switzerland, 2018; Volume 1, pp. 1-29. Available online: https:/ / doi.org/10.1007/978-3-319-90481-8_1 (accessed on 23 April 2021).

28. Tagirov, L.R.; Kupriyanov, M.Y.; Kushnir, V.N.; Sidorenko, A. Basic superconducting spin valves. In Functional Nanostructures and Metamaterials for Superconducting Spintronics (From Superconducting Qubits to Self-Organized Nanostructures), Springer Series Nano Science and Technology; Sidorenko, A., Ed.; Springer International Publishing: Cham, Switzerland, 2018 ; Volume 1, pp. 31-47. Available online: https://doi.org/10.1007/978-3-319-90481-8_2 (accessed on 23 April 2021).

29. Bakurskiy, S.; Kupriyanov, M.; Klenov, N.V.; Soloviev, I.; Schegolev, A.; Morari, R.; Khaydukov, Y.; Sidorenko, A.S. Controlling the proximity effect in a $\mathrm{Co} / \mathrm{Nb}$ multilayer; the properties of electronic transport. Beilstein J. Nanotechnol. 2020, 11, 1336-1345. [CrossRef] [PubMed]

30. Tagirov, L.R. Low-field superconducting spin switch based on a superconductor/ferromagnet multilayer. Phys. Rev. Lett. 1999, 83, 2058-2061. [CrossRef]

31. Bergeret, F.S.; Volkov, A.F.; Efetov, K.B. Long-range proximity effects in superconductor-ferromagnet structures. Phys. Rev. Lett. 2001, 86, 4096-4099. [CrossRef]

32. Pannetier, B.; Rodts, S.; Genicon, J.L.; Otani, Y.; Nozières, J.P. Macroscopic Quantum Phenomena and Coherence in Superconducting Networks Chapter (Nucleation of Superconductivity in a Thin Film in a Spatially Modulated Magnetic Field); World Scientific: Singapore, 1995; pp. 17-24.

33. Sonin, E.B. Superconductivity suppression (weak link) by domain wall in bilayer superconductor-ferromagnet film. Sov. Tech Phys. Lett. 1988, 14, 1640-1644.

34. Aladyshkin, A.Y.; Buzdin, A.I.; Fraerman, A.A.; Mel'nikov, A.S.; Ryzhov, D.A.; Sokolov, A.V. Domain-wall superconductivity in hybrid superconductor-ferromagnetic structures. Phys. Rev. B 2003, 68, 184508. [CrossRef]

35. Aladyshkin, A.Y.; Moshchalkov, V.V. Thin-film superconductor-ferromagnet hybrids: Competition between nucleation of superconductivity at domain walls and domain's centers. Phys. Rev. B 2006, 74, 064503. [CrossRef]

36. Houzet, M.; Buzdin, A.I. Theory of domain-wall superconductivity in superconductor/ferromagnetic bilayers. Phys. Rev. B 2006, 74, 214507. [CrossRef]

37. Gillings, W.; Aladyshkin, A.Y.; Silhanek, A.V.; Moshchalkov, V.V. Magnetic confinement of the superconducting condensate in superconductor-ferromagnet composites. Phys. Rev. B 2007, 76, 060503. [CrossRef]

38. Aladyshkin, A.Y.; Mel'nikov, A.S.; Nefedov, I.M.; Savinov, D.A.; Silaev, M.A.; Shereshevskii, I.A. Hybridization and interference effects for localized superconducting states in strong magnetic field. Phys. Rev. B 2012, 85, 184528. [CrossRef]

39. Aladyshkin, A.Y.; Fritzsche, J.; Werner, R.; Kramer, R.B.G.; Guénon, S.; Kleiner, R.; Koelle, D.; Moshchalkov, V.V. Crossover between different regimes of inhomogeneous superconductivity in planar superconductor-ferromagnet hybrids. Phys. Rev. $B$ 2011, 84, 094523. [CrossRef]

40. Werner, R.; Aladyshkin, A.Y.; Guénon, S.; Fritzsche, J.; Nefedov, I.M.; Moshchalkov, V.V.; Kleiner, R.; Koelle, D. Domain-wall and reverse-domain superconducting states of a Pb thin-film bridge on a ferromagnetic $\mathrm{BaFe}_{12} \mathrm{O}_{19}$ single crystal. Phys. Rev. B 2011, 84, 020505. [CrossRef]

41. Trezza, M.; Cirillo, C.; Vorobjeva, A.I.; Outkina, E.A.; Prischepa, S.L.; Attanasio, C. Vortex matching effects in Nb thin films due to Ni nanopillars embedded in anodic aluminum oxide substrates. Supercond. Sci. Technol. 2013, 26, 035001. [CrossRef]

42. Stellhorn, A.; Sarkar, A.; Kentzinger, E.; Barthel, J.; Di Bernardo, A.; Nandi, S.; Zakalek, P.; Schubert, J.; Brückel, T. Tailoring superconducting states in superconductor-ferromagnet hybrids. New J. Phys. 2020, 22, 093001. [CrossRef]

43. Prischepa, S.L.; Cirillo, C.; Kushnir, V.N.; Ilyina, E.A.; Salvato, M.; Attanasio, C. Effect of geometrical symmetry on the angular dependence of the critical magnetic field in superconductor/normal metal multilayers. Phys. Rev. B 2005, 72, 024535. [CrossRef]

44. Kushnir, V.N.; Prischepa, S.L.; Cirillo, C.; Della Rocca, S.L.; Angrisani Armenio, A.; Maritato, L.; Salvato, M.; Attanasio, C. Nucleation of superconductivity in finite metallic multilayers: Effect of the symmetry. Eur. Phys. J. B 2004, 41, 439-444. [CrossRef]

45. Kushnir, V.N.; Prischepa, S.L.; Della Rocca, M.L.; Salvato, M.; Attanasio, C. Effect of symmetry on the resistive characteristics of proximity coupled metallic multilayers. Phys. Rev. B 2003, 68, 212505. [CrossRef]

46. Autler, S.H. Fluxoid pinning in superconductors by a periodic array of magnetic particles. J. Low Temp. Phys. 1972, 9, 241-253. [CrossRef]

47. Milošević, M.V.; Peeters, F.M. Field-enhanced critical parameters in magnetically nanostructured superconductors. Europhys. Lett. 2005, 70, 670-676. [CrossRef]

48. Gheorghe, D.G.; Wijngaarden, R.J.; Gillijns, W.; Silhanek, A.V.; Moshchalkov, V.V. Magnetic flux patterns in superconductors deposited on a lattice of magnetic dots: A magneto-optical imaging study. Phys. Rev. B 2008, 77, 054502. [CrossRef]

49. Prischepa, S.L.; Kupriyanov, M.Y.; Cirillo, C.; Attanasio, C. Magnetic memory effect in type-II superconductor/ferromagnet bilayers. Supercond. Sci. Technol. 2014, 27, 055024. [CrossRef]

50. Trezza, M.; Cirillo, C.; Dolgiy, A.L.; Redko, S.V.; Bondarenko, V.P.; Andreyenka, A.V.; Danilyuk, A.L.; Prischepa, S.L.; Attanasio, C. Change of the topology of a superconducting thin film electromagnetically coupled with an array of ferromagnetic nanowires. Supercond. Sci. Technol. 2016, 29, 015011. [CrossRef] 
51. Kushnir, V.N.; Ilyina, E.A.; Prischepa, S.L.; Cirillo, C.; Attanasio, C. Role of the external surfaces on the superconducting properties on superconductor/normal metal trilayers. Superlattices Microstruct. 2008, 43, 86-92. [CrossRef]

52. Cirillo, C.; Ilyina, E.A.; Attanasio, C. Static and dynamic properties of the vortex lattice in superconductor/weak ferromagnet bilayers. Supercond. Sci. Technol. 2011, 24, 024017. [CrossRef]

53. Khaire, T.S.; Pratt, W.P., Jr.; Birge, N.O. Critical current behavior in Josephson junctions with the weak ferromagnet PdNi. Phys. Rev. B 2009, 79, 094523. [CrossRef]

54. Samson, Y.; Marty, A.; Hoffman, R.; Gehanno, V.; Gilles, B. Magnetic domains in thin fils with perpendicular anisotropy: An extensive study. J. Appl. Phys. 1999, 85, 4604-4606. [CrossRef]

55. Yang, Y.; Chen, J.S.; Chow, G.M. Highly chemical ordered $L 1_{1}$ CoPt (111) films with perpendicular anisotropy grown on glass substrates. J. Appl. Phys. 2011, 109, 7. [CrossRef]

56. Kawada, Y.; Onose, M.; Tojo, R.; Komine, T.; Sugita, R. Magnetic domain structure in thin CoPt perpendicular magnetic anisotropy films. EPJ Web Conf. 2013, 40, 07002. [CrossRef]

57. Zhang, D.; Shaw, J.M.; Smith, D.J.; McCartney, M.R. Domain structure and perpendicular magnetic anisotropy in CoFe/Pd multilayers using off-axis electron holography. J. Magn. Magn. Mater. 2015, 388, 16-21. [CrossRef]

58. Skaugen, A.; Murray, P.; Laurson, L. Analytical computation of the demagnetizing energy of thin film domain walls. Phys. Rev. B 2019, 100, 094440. [CrossRef]

59. Landau, L.D.; Lifshitz, E.M. Electrodynamics of Continuous Media: Volume 8 (Course of Theoretical Physics S), 2nd ed.; Institute of Physical Problems of the USSR Academy of Sciences: Moscow, Russia, 1984; 460p.

60. Malozemoff, A.P.; Slonczewski, J.C. Magnetic Domain Walls in Bubble Materials; Academic Press: Cambridge, MA, USA, 1979.

61. Veshchunov, I.S.; Oboznov, V.A.; Rossolenko, A.N.; Prokofiev, A.S.; Vinnikov, I.Y.; Rusanov, A.Y.; Matveev, D.V. Observation of the magnetic domain structure in $\mathrm{Cu}_{0.47} \mathrm{Ni}_{0.53}$ thin films at low temperatures. JETP Lett. 2008, 88, 758-761. [CrossRef]

62. Sonin, E.B.; Felner, I. Spontaneous vortex phase in a superconducting weak ferromagnet. Phys. Rev. B 1998, 57, R14000-R14003. [CrossRef]

63. Bespyatykh, Y.I.; Wasilevski, W. The spontaneous formation of a vortex structure in a type II superconductor-ferromagnet bilayer. Phys. Solid State 2001, 43, 224-230. [CrossRef]

64. Usadel, K.D. Generalized diffusion equation for superconducting alloys. Phys. Rev. Lett. 1970, 25, 507-509. [CrossRef]

65. Maki, K. The behavior of superconducting thin films in the presence of magnetic fields and currents. Prog. Theor. Phys. 1964, 31, 731-741. [CrossRef]

66. Abramovitz, M.; Stegun, I.A. (Eds.) Handbook of Mathematical Functions with Formulas, Graphs, and Mathematical Tables; Applied Mathematics Series 55; National Bureau of Standards: Gaithersburg, MD, USA, 1964; 1046p.

67. de Gennes, P.G. Superconductivity of Metals and Alloys; W.A. Benjamin, Inc.: San Francisco, CA, USA, 1966; p. 274.

68. Yakubovich, V.A.; Starzhinskii, V.M. Linear Differential Equations with Periodic Coefficients; John Wiley \& Sons: Hoboken, NJ, USA, 1975; Volume 1, p. 386.

69. Abrikosov, A.A. Fundamentals of the Theory of Metals; North-Holland: Amsterdam, The Netherlands, 1988; 630p.

70. Campbell, A.M.; Evetts, J.E. Critical Currents in Superconductors; Taylor and Francis: London, UK, 1972; 243p.

71. Bogoliubov, N.N.; Mitropolsky, Y.A. Asymptotic Methods in the Theory of Nonlinear Oscillations; Gordon and Breach: London, UK, $1961 ; 548 \mathrm{p}$ 